\title{
Optimal Guaranteed Cost Control of a Class of Discrete-Time Nonlinear Systems with Markovian Switching and Mode-Dependent Mixed Time Delays
}

\author{
Yurong Liu, ${ }^{1}$ Hamid Reza Karimi, ${ }^{2}$ and Xiaohui Liu $^{3}$ \\ ${ }^{1}$ Department of Mathematics, Yangzhou University, Yangzhou 225002, China \\ ${ }^{2}$ Department of Engineering, Faculty of Engineering and Science, University of Agder, 4898 Grimstad, Norway \\ ${ }^{3}$ School of Computer Science and Technology, Nanjing University of Science and Technology, Nanjing 210094, China \\ Correspondence should be addressed to Yurong Liu; liuyurong@gmail.com
}

Received 8 May 2013; Accepted 8 June 2013

Academic Editor: Zidong Wang

Copyright ( 2013 Yurong Liu et al. This is an open access article distributed under the Creative Commons Attribution License, which permits unrestricted use, distribution, and reproduction in any medium, provided the original work is properly cited.

\begin{abstract}
The guaranteed cost control problem is investigated for a class of nonlinear discrete-time systems with Markovian jumping parameters and mixed time delays. The mixed time delays involved consist of both the mode-dependent discrete delay and the distributed delay with mode-dependent lower bound. The associated cost function is of a quadratic summation form over the infinite horizon. The nonlinear functions are assumed to satisfy sector-bounded conditions. By introducing new LyapunovKrasovskii functionals and developing some new analysis techniques, sufficient conditions for the existence of guaranteed cost controllers are derived with respect to the given cost function. Moreover, a convex optimization approach is applied to search for the optimal guaranteed cost controller by minimizing the guaranteed cost of the closed-loop system. Numerical simulation is further carried out to demonstrate the effectiveness of the proposed methods.
\end{abstract}

\section{Introduction}

In the past decades, the control problems for the linear or nonlinear systems have attracted considerable research interest and significant advances on this topic have been made; see, for example, [1-15] and the references therein. It is well known that the time delay in feedback control can be caused by physical properties of control equipments, measurements of system responses, and data processing, calculating and executing control forces, and so forth. The time delay in feedback control may not only deteriorate the performance of controlled systems but also destabilize the controlled systems. There have been a lot of reports on the dynamics analysis of time delay feedback controlled systems. Various sufficient conditions, either delay dependent or delay independent, have been proposed to guarantee the stability for the delayed systems; see, for example, [2, 9, 12, 13] for some recent publications.
On the other hand, a great deal of attention has recently been devoted to the study of Markovian jump systems. This class of systems can be modeled with variable structure subject to random abrupt changes resulting from the occurrence of some inner discrete events in the system such as failures and repairs of machine in manufacturing systems, random failures and repairs of the components, changes in the interconnections of subsystems, sudden environment changes, and so on. Recently, stability and control problems for Markovian jump systems have been extensively investigated; see, for example, $[16,17]$ and the references therein.

It is also noted that in practical applications, the choice of control policy depends upon the optimization of some preassigned performance criterion. When designing a controller for a real system, it is often desirable to make the controlled system not only stable but also guarantee an adequate level of performance. To deal with such control 
problems, the so-called guaranteed cost control approach was first introduced by Chang and Peng [2]. The objective of this approach is to establish an upper bound on a given performance index so that the system performance degradation incurred by the uncertainties is guaranteed to be less than this bound. For guaranteed cost control, a great number of results on this topic have been reported in the literature and various approaches have been proposed. For example, in [18], notion of the quadratic guaranteed cost control was introduced to allow for a quadratic performance index and a Riccati equation approach was presented for designing quadratic guaranteed cost controllers, where the system was delay-free. The authors in [19] extended the Riccati equation approach given in [18] to uncertain delayed systems and proposed a guaranteed cost controller design method by solving a certain parameter-dependent Riccati equation. In [15], an LMI approach [20] was proposed to deal with the guaranteed cost control problem for a class of linear time delay systems with time-varying normbounded parameter uncertainty, and a sufficient condition for the existence of memoryless state-feedback guaranteed cost controllers was derived. In [21], the solutions to the guaranteed cost control problem via state-feedback are presented for a class of uncertain Markovian jump systems with mode-dependent delays in LMI framework, and the delay-dependent/independent sufficient conditions for the existence of guaranteed cost state-feedback controllers have been derived.

Based on LMI approach, [22] considered the robust guaranteed cost control problem for uncertain linear discretetime systems subject to actuator saturation, where the saturation nonlinearity was transformed into a convex polytope of linear systems, and then this problem was formulated into a convex optimization problem with constraints given by a set of LMIs. Very recently, the filtering problems have been investigated for discrete-time nonlinear stochastic systems with network-induced phenomena in $[7,8,23,24]$. As far as we know, however, little research has been focused on the guaranteed cost control problem for discrete-time systems with distributed time delay and Markovian jumping parameters.

In this paper, we consider the guaranteed cost control problem for a class of nonlinear discrete-time systems with Markovian jumping parameters and mixed time delays. The mixed time delays involved consist of both the modedependent discrete delay and the infinite distributed delay with mode-dependent lower bound. The relevant cost function is chosen as a quadratic summation form over the infinite horizon. The nonlinear functions are assumed to satisfy sector-bounded conditions. By constructing novel Lyapunov-Krasovskii functionals and employing some new analysis techniques, sufficient conditions for the existence of guaranteed cost controllers are derived with respect to the given cost function. In addition, a convex optimization approach is applied to search for the optimal guaranteed cost controller by minimizing the guaranteed cost of the closed-loop system. Finally, a numerical example is presented to demonstrate the effectiveness of the proposed methods.
Notations. Throughout this paper, $\mathbb{R}^{n}$ and $\mathbb{R}^{n \times m}$ denote, respectively, the $n$-dimensional Euclidean space and the set of all $n \times m$ real matrices; $\mathbb{N}^{-}$stands for the set of all the negative integers and zero. The superscript " $T$ " denotes matrix transposition. The notation $X \geq Y$ (resp., $X>$ $Y$ ), where $X$ and $Y$ are symmetric matrices, means that $X-Y$ is positive semidefinite (resp., positive definite). $\operatorname{diag}\{\cdots\}$ stands for a block-diagonal matrix, $I$ is the identity matrix with compatible dimension, and $|\cdot|$ denotes the Euclidean norm in $\mathbb{R}^{n}$. If $A$ is a square matrix, $\lambda_{\text {max }}(A)$ (resp., $\left.\lambda_{\text {min }}(A)\right)$ denotes the largest (resp., smallest) eigenvalue of $A$, and $\operatorname{Tr}(A)$ denotes the trace of $A$. In symmetric block matrices, an asterisk " $*$ " is used to represent a term that is induced by symmetry. $\mathbb{E}[x]$ and $\mathbb{E}[x \mid y]$ will, respectively, mean the expectation of $x$ and the expectation of $x$ conditional on $y$. Matrices, if their dimensions are not explicitly stated, are assumed to be compatible for algebraic operations.

\section{Problem Formulation}

Let $r(k)(k \geq 0)$ be a Markov chain taking values in a finite state space $\mathcal{N}=\left\{1,2, \ldots, n_{0}\right\}$ with probability transition matrix $\Pi=\left(\pi_{i j}\right)_{n_{0} \times n_{0}}$ given by

$$
\operatorname{Pr}\{r(k+1)=j \mid r(k)=i\}=\pi_{i j}, \quad \forall i, j \in \mathcal{N},
$$

where $\pi_{i j} \geq 0(i, j \in \mathscr{N})$ is the transition probability from $i$ to $j$ and $\sum_{j=1}^{n_{0}} \pi_{i j}=1$, for all $i \in N$.

Consider a discrete-time nonlinear system with $n_{0}$ modes described by the following dynamical equation:

$$
\begin{aligned}
& x(k+1)= A(r(k)) x(k)+B(r(k)) f(x(k)) \\
&+C(r(k)) g\left(x\left(k-\tau_{1, r(k)}\right)\right)+D(r(k)) \\
& \times \sum_{m=\tau_{2, r(k)}}^{+\infty} \mu_{m} h(x(k-m))+E(r(k)) u(k), \\
& x(m)=\phi(m) \quad \text { for } m \in \mathbb{N}^{-}, r(0)=r_{0},
\end{aligned}
$$

where $x(k) \in \mathbb{R}^{n}$ is the state vector; for $r(k)=i \in \mathcal{N}$, $A(r(k)) \in \mathbb{R}^{n \times n}, B(r(k)) \in \mathbb{R}^{n \times n}, C(r(k)) \in \mathbb{R}^{n \times n}, D(r(k)) \in$ $\mathbb{R}^{n \times n} \in \mathbb{R}^{n \times n}$, and $E(r(k)) \in \mathbb{R}^{n \times q}$ are known constant matrices; $f(\cdot), g(\cdot)$ and $h(\cdot): \mathbb{R}^{n} \rightarrow \mathbb{R}^{n}$ are nonlinear vector functions; $\mu_{m}(m=1,2, \ldots)$ are scalar constants; $u(k) \in \mathbb{R}^{q}$ is the control input; $\tau_{1, r(k)}$ stands for the modedependent discrete time-delay while $\tau_{2, r(k)} \geq 0$ describes a mode-dependent lower bound for the distributed time-delay; $\phi: \mathbb{N}^{-} \rightarrow \mathbb{R}^{n}$ is the initial value; and $r(0)=r_{0}$ is the initial mode of the Markov chain. 
For nonlinear vector functions $f, g, h$, we assume that

$$
\begin{array}{ll}
\left(f(x)-F_{1} x\right)^{T}\left(f(x)-F_{2} x\right) \leq 0, & \forall x \in \mathbb{R}^{n}, \\
\left(g(x)-G_{1} x\right)^{T}\left(g(x)-G_{2} x\right) \leq 0, & \forall x \in \mathbb{R}^{n}, \\
\left(h(x)-H_{1} x\right)^{T}\left(h(x)-H_{2} x\right) \leq 0, & \forall x \in \mathbb{R}^{n},
\end{array}
$$

where $F_{1}, F_{2}, G_{1}, G_{2}, H_{1}$, and $H_{2} \in \mathbb{R}^{(n \times n)}$ are known constant matrices.

Remark 1. The conditions (2) are quite general, and such a description, compared with the usual Lipschitz condition, is very helpful for using LMI-based approach to reduce the possible conservatism. The similar form of the conditions has been used, for example, by the authors in [10].

Remark 2. It is not difficult to verify that the conditions (2) imply that $f(0)=g(0)=h(0)=0$, and $x=0$ is therefore an equilibrium point.

The cost function associated with system (2a) and (2b) is

$$
\begin{aligned}
J=\mathbb{E}\left[\sum _ { k = 0 } ^ { \infty } \left(x^{T}(k) R_{1}(r(k)) x(k)\right.\right. \\
\left.\left.\quad+u^{T}(t) R_{2}(r(k)) u(k)\right) \mid \phi(k), r_{0}\right],
\end{aligned}
$$

where $R_{1}(i)>0$ and $R_{2}(i)>0$, for all $i \in \mathcal{N}$.

Now, consider the following state-feedback control law $u(k)=K(r(k)) x(k)$, where $K(i) \in \mathbb{R}^{q \times n}(i \in \mathcal{N})$ are controller gains to be designed. Then, the closed-loop system can be given as follows:

$$
\begin{aligned}
x(k+1)= & A_{K}(r(k)) x(k)+B(r(k)) f(x(k)) \\
& +C(r(k)) g\left(x\left(k-\tau_{1, r(k)}\right)\right) \\
& +D(r(k)) \sum_{m=\tau_{2, r(k)}}^{+\infty} \mu_{m} h(x(k-m)), \\
x(m)= & \phi(m) \quad \text { for } m \in \mathbb{N}^{-}, r(0)=r_{0},
\end{aligned}
$$

where $A_{K}(r(k))=A(r(t))+E(r(k)) K(r(k))$.

Definition 3. System (2a) and (2b) with $u(k) \equiv 0$ is said to be asymptotically stable in mean square if, for any solution $x(k)$ of system (2a) and (2b), the following holds:

$$
\lim _{k \rightarrow \infty} \mathbb{E}\left[|x(k)|^{2}\right]=0
$$

Definition 4. Consider the system (2a) and (2b). If there exists a state-feedback control law $u(k)$ and a positive number $\gamma$ such that the closed-loop system (5a) and (5b) is asymptotically stable in mean square and the resulting cost function satisfies

$$
J \leq \gamma
$$

then $\gamma$ is said to be a guaranteed cost and $u(k)$ is said to be a guaranteed cost controller for the system (2a) and (2b).

The objective of this paper is to develop a procedure to design a memoryless state-feedback guaranteed cost controller $u(k)=K(r(k)) x(k)$, which achieves as small value of $\gamma$ as possible.

Assumption 5. Constant $\mu_{m} \geq 0$ satisfies the following convergent conditions:

$$
\sum_{m=1}^{+\infty} \mu_{m}<+\infty, \quad \sum_{m=1}^{+\infty} m \mu_{m}<+\infty
$$

Remark 6. Assumption 5 makes sense as they guarantee that the term $D(r(k)) \sum_{m=\tau_{2, r(k)}}^{+\infty} \mu_{m} h(x(k-m))$ in (2a) is convergent, which is necessary for the subsequent analysis.

\section{Main Results and Proofs}

We first introduce some lemmas to be used in deriving our results.

Lemma 7 (see [25]). Let $M \in \mathbb{R}^{n \times n}$ be a positive semi definite matrix, $\mathbf{x}_{i} \in \mathbb{R}^{n}$ and $a_{i} \geq 0(i=1,2, \ldots)$. If the series concerned are convergent, the following inequality holds:

$$
\left(\sum_{i=1}^{+\infty} a_{i} \mathbf{x}_{i}\right)^{T} M\left(\sum_{i=1}^{+\infty} a_{i} \mathbf{x}_{i}\right) \leq\left(\sum_{i=1}^{+\infty} a_{i}\right) \sum_{i=1}^{+\infty} a_{i} \mathbf{x}_{i}^{T} M \mathbf{x}_{i}
$$

Lemma 8 (see [10]). Assume that nonlinear function $\hbar(\cdot)$ : $\mathbb{R}^{n} \rightarrow \mathbb{R}^{n}$ satisfies

$$
\left(\hbar(x)-U_{1} x\right)^{T}\left(\hbar(x)-U_{2} x\right) \leq 0, \quad \forall x \in \mathbb{R}^{n}
$$

with $U_{1}$ and $U_{2}$ being constant matrices. Then, the following matrix inequality holds:

$$
\left[\begin{array}{c}
x \\
\hbar(x)
\end{array}\right]^{T}\left[\begin{array}{cc}
\breve{U}_{1} & -\breve{U}_{2} \\
-\breve{U}_{2}^{T} & I
\end{array}\right]\left[\begin{array}{c}
x \\
\hbar(x)
\end{array}\right] \leq 0
$$


or

$$
x^{T} \breve{U}_{1} x-2 x^{T} \breve{U}_{2} \hbar(x)+\hbar^{T}(x) \hbar(x) \leq 0,
$$

where $\breve{U}_{1}=\left(U_{1}^{T} U_{2}+U_{2}^{T} U_{1}\right) / 2$ and $\breve{U}_{2}=\left(U_{1}^{T}+U_{2}^{T}\right) / 2$.

Proof. It can be verified by simple matrix operations.

Lemma 9 (Schur complement [20]). Given constant matrices $\Omega_{1}, \Omega_{2}, \Omega_{3}$ where $\Omega_{1}=\Omega_{1}^{T}$ and $\Omega_{2}>0$, then

$$
\Omega_{1}+\Omega_{3}^{T} \Omega_{2}^{-1} \Omega_{3}<0
$$

if and only if

$$
\left[\begin{array}{cc}
\Omega_{1} & \Omega_{3}^{T} \\
\Omega_{3} & -\Omega_{2}
\end{array}\right]<0 \quad \text { or } \quad\left[\begin{array}{cc}
-\Omega_{2} & \Omega_{3} \\
\Omega_{3}^{T} & \Omega_{1}
\end{array}\right]<0
$$

Hereafter, one denote $\bar{\tau}_{1}=\max _{1 \leq j \leq n_{0}}\left\{\tau_{1, j}\right\}, \bar{\tau}_{2}=$ $\max _{1 \leq j \leq n_{0}}\left\{\tau_{2, j}\right\}, \underline{\tau}_{1}=\min _{1 \leq j \leq n_{0}}\left\{\tau_{1, j}\right\}, \underline{\tau}_{2}=\min _{1 \leq j \leq n_{0}}\left\{\tau_{2, j}\right\}$, and $\underline{\pi}=\min _{1 \leq i \leq n_{0}}\left\{\left|\pi_{i i}\right|\right\}$.

One also denotes

$$
\begin{gathered}
\breve{F}_{1}=\frac{\left(F_{1}^{T} F_{2}+F_{2}^{T} F_{1}\right)}{2}, \quad \breve{F}_{2}=\frac{\left(F_{1}^{T}+F_{2}^{T}\right)}{2}, \\
\breve{G}_{1}=\frac{\left(G_{1}^{T} G_{2}+G_{2}^{T} G_{1}\right)}{2}, \quad \breve{G}_{2}=\frac{\left(G_{1}^{T}+G_{2}^{T}\right)}{2}, \\
\breve{H}_{1}=\frac{\left(H_{1}^{T} H_{2}+H_{2}^{T} H_{1}\right)}{2}, \quad \breve{H}_{2}=\frac{\left(H_{1}^{T}+H_{2}^{T}\right)}{2}, \\
\sigma_{m}=\sum_{l=m}^{+\infty} \mu_{l}, \quad \widehat{\mu}=\max \left\{\mu_{m} \mid \underline{\tau}_{2} \leq m \leq \bar{\tau}_{2}-1\right\} .
\end{gathered}
$$

The following is a sufficient condition for the existence of state-feedback guaranteed cost control laws for the system (2a) and (2b).

Theorem 10. Given a state-feedback controller $u(k)=$ $K(r(k)) x(k)$. If there exist a set of positive definite matrices $P_{i}(i \in \mathcal{N})$, and two positive definite matrices $Q_{1}$ and $Q_{2}$ such that the LMIs (17) hold, then $u(k)=K(r(k)) x(k)$ is a guaranteed cost controller for the system (2a) and (2b), and the cost function satisfies the following bound:

$$
J \leq x^{T}(0) P_{r_{0}} x(0)+\sum_{v=-\tau_{1, r_{0}}}^{-1} g^{T}(x(v)) Q_{1} g(x(v))
$$

$$
\begin{aligned}
& +(1-\underline{\pi}) \sum_{l=\underline{\tau}_{1}}^{\bar{\tau}_{1}-1} \sum_{v=-l}^{-1} g^{T}(x(v)) Q_{1} g(x(v)) \\
& +\sum_{\iota=\tau_{2, r_{0}}}^{+\infty} \mu_{\iota} \sum_{v=-\iota}^{-1} h^{T}(x(v)) Q_{2} h(x(v)) \\
& +(1-\underline{\pi}) \widehat{\mu} \sum_{s=\underline{\tau}_{2}}^{\bar{\tau}_{2}-1} \sum_{\iota=1}^{s-1} \sum_{v=-\iota}^{-1} h^{T}(x(v)) Q_{2} h(x(v)),
\end{aligned}
$$

$\Phi(i)$

$$
\begin{aligned}
& =\left[\begin{array}{ccc}
-P_{i}-\Xi+R_{1}(i)+K^{T}(i) R_{2} K(i) & \Theta & A_{K}^{T}(i) \bar{P}_{i} \\
\Theta^{T} & \Upsilon(i) & \Sigma^{T}(i) \bar{P}_{i} \\
\bar{P}_{i} A_{K}(i) & \bar{P}_{i} \Sigma(i) & -\bar{P}_{i}
\end{array}\right] \\
& <0, \quad i \in \mathcal{N},
\end{aligned}
$$

where

$$
\begin{aligned}
& \Xi=\breve{F}_{1}+\breve{G}_{1}+\breve{H}_{1}, \quad \bar{P}_{i}=\sum_{j=1}^{n_{0}} \pi_{i j} P_{j}, \\
& \Theta=\left[\begin{array}{lllll}
\breve{F}_{2} & \breve{G}_{2} & 0 & \breve{H}_{2} & 0
\end{array}\right], \\
& \Sigma(i)=\left[\begin{array}{lllll}
B(i) & 0 & C(i) & 0 & D(i)
\end{array}\right] \\
& \Upsilon(i)=\operatorname{diag}\left\{-I, \alpha_{1} Q_{1}-I,\right. \\
& \left.-Q_{1}, \alpha_{2} Q_{2}-I,-\frac{1}{\sigma_{\tau_{2, i}}} Q_{2}\right\}
\end{aligned}
$$

with

$$
\begin{aligned}
& \alpha_{1}=(1-\underline{\pi})\left(\bar{\tau}_{1}-\underline{\tau}_{1}\right)+1, \\
& \alpha_{2}=\sigma_{\underline{\tau}_{2}}+\frac{1}{2} \widehat{\mu}(1-\underline{\pi})\left(\bar{\tau}_{2}-\underline{\tau}_{2}\right)\left(\bar{\tau}_{2}+\underline{\tau}_{2}-3\right) .
\end{aligned}
$$

Proof. For convenience, we denote

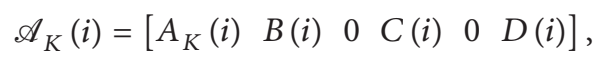

$$
\begin{aligned}
& \xi\left(\mathbf{x}_{k}, i\right)=\left[\begin{array}{llllll}
x^{T}(k) & f^{T}(x(k)) & g^{T}(x(k)) & g^{T}\left(x\left(k-\tau_{1, r(k)}\right)\right) & h^{T}(x(k)) & \sum_{m=\tau_{2, i}}^{+\infty} \mu_{m} h^{T}(x(k-m))
\end{array}\right]^{T} .
\end{aligned}
$$


By Lemma 9, the inequality (17) is equivalent to

$$
\bar{\Phi}(i)+\mathscr{A}_{K}^{T}(i) \bar{P}_{i} \mathscr{A}_{K}(i)<0
$$

where

$$
\bar{\Phi}(i)=\left[\begin{array}{cc}
-P_{i}-\Xi+R_{1}(i)+K^{T}(i) R_{2}(i) K(i) & \Theta \\
\Theta^{T} & \Upsilon(i)
\end{array}\right] .
$$

Define $\mathbf{x}_{k}: \mathbb{N}^{-} \rightarrow \mathbb{R}^{n}$ by $\mathbf{x}_{k}(m)=x(k+m)$ for $m \in \mathbb{N}^{-}$. To proceed the stability analysis, we construct the following Lyapunov-Krasovskii functional for the system (5a) and (5b):

$$
V\left(\mathbf{x}_{k}, k, r(k)\right)=\sum_{i=1}^{5} V_{i}\left(\mathbf{x}_{k}, k, r(k)\right)
$$

where

$V_{1}\left(\mathbf{x}_{k}, k, r(k)\right)=x^{T}(k) P_{r(k)} x(k)$,

$V_{2}\left(\mathbf{x}_{k}, k, r(k)\right)=\sum_{v=k-\tau_{1, r(k)}}^{k-1} g^{T}(x(v)) Q_{1} g(x(v))$,

$V_{3}\left(\mathbf{x}_{k}, k, r(k)\right)=(1-\underline{\pi}) \sum_{l=\underline{\tau}_{1}}^{\bar{\tau}_{1}-1} \sum_{v=k-l}^{k-1} g^{T}(x(v)) Q_{1} g(x(v))$,

$V_{4}\left(\mathbf{x}_{k}, k, r(k)\right)=\sum_{\iota=\tau_{2, r(k)}}^{+\infty} \mu_{\iota} \sum_{v=k-\iota}^{k-1} h^{T}(x(v)) Q_{2} h(x(v))$,

$V_{5}\left(\mathbf{x}_{k}, k, r(k)\right)=(1-\underline{\pi}) \widehat{\mu} \sum_{s=\underline{\tau}_{2}}^{\bar{\tau}_{2}-1} \sum_{l=1}^{s-1} \sum_{v=k-\iota}^{k-1} h^{T}(x(v)) Q_{2} h(x(v))$.

For $i \in \mathcal{N}$, associated with the closed-loop system (5a) and (5b) we can carry out the following computation:

$$
\begin{aligned}
\mathbb{E} & {\left[V_{1}\left(\mathbf{x}_{k+1}, k+1, r(k+1)\right) \mid \mathbf{x}_{k}, r(k)=i\right]-V_{1}\left(\mathbf{x}_{k}, k, i\right) } \\
= & \sum_{j=1}^{n_{0}} \pi_{i j} x^{T}(k+1) P_{j} x(k+1)-x^{T}(k) P_{i} x(k) \\
= & \xi^{T}\left(\mathbf{x}_{\mathbf{k}}, i\right) \mathscr{A}_{K}^{T}(i) \bar{P}_{i} \mathscr{A}_{K}(i) \xi\left(\mathbf{x}_{\mathbf{k}}, i\right)-x^{T}(k) P_{i} x(k) ; \\
\mathbb{E} & {\left[V_{2}\left(\mathbf{x}_{k+1}, k+1, r(k+1)\right) \mid \mathbf{x}_{k}, r(k)=i\right]-V_{2}\left(\mathbf{x}_{k}, k, i\right) } \\
= & \sum_{j=1}^{n_{0}} \pi_{i j} \sum_{v=k-\tau_{1, j}+1}^{k} g^{T}(x(v)) Q_{1} g(x(v)) \\
& -\sum_{v=k-\tau_{1, i}}^{k-1} g^{T}(x(v)) Q_{1} g(x(v))
\end{aligned}
$$

$$
\begin{aligned}
= & \sum_{j=1}^{n_{0}} \pi_{i j} g^{T}(x(k)) Q_{1} g(x(k)) \\
& -g^{T}\left(x\left(k-\tau_{1, i}\right)\right) Q_{1} g\left(x\left(k-\tau_{1, i}\right)\right)
\end{aligned}
$$$$
+\sum_{j=1}^{n_{0}} \pi_{i j} \sum_{v=k-\tau_{1, j}+1}^{k-1} g^{T}(x(v)) Q_{1} g(x(v))
$$$$
-\sum_{v=k-\tau_{1, i}+1}^{k-1} g^{T}(x(v)) Q_{1} g(x(v))
$$$$
=g^{T}(x(k)) Q_{1} g(x(k))-g^{T}\left(x\left(k-\tau_{1, i}\right)\right)
$$$$
\times Q_{1} g\left(x\left(k-\tau_{1, i}\right)\right)
$$$$
+\sum_{j \neq i} \pi_{i j}\left(\sum_{v=k-\tau_{1, j}+1}^{k-1} g^{T}(x(v)) Q_{1} g(x(v))\right.
$$

$$
\left.-\sum_{v=k-\tau_{1, i}+1}^{k-1} g^{T}(x(v)) Q_{1} g(x(v))\right)
$$$$
\leq g^{T}(x(k)) Q_{1} g(x(k))-g^{T}\left(x\left(k-\tau_{1, i}\right)\right) Q_{1} g\left(x\left(k-\tau_{1, i}\right)\right)
$$$$
+\sum_{j \neq i} \pi_{i j} \sum_{v=k-\bar{\tau}_{1}+1}^{k-\underline{\tau}_{1}} g^{T}(x(v)) Q_{1} g(x(v))
$$$$
\leq g^{T}(x(k)) Q_{1} g(x(k))-g^{T}\left(x\left(k-\tau_{1, i}\right)\right) Q_{1} g\left(x\left(k-\tau_{1, i}\right)\right)
$$$$
+(1-\underline{\pi}) \sum_{v=k-\bar{\tau}_{1}+1}^{k-\underline{\tau}_{1}} g^{T}(x(v)) Q_{1} g(x(v)) ;
$$$$
\mathbb{E}\left[V_{3}\left(\mathbf{x}_{k+1}, k+1, r(k+1)\right) \mid \mathbf{x}_{k}, r(k)=i\right]-V_{3}\left(\mathbf{x}_{k}, k, i\right)
$$

$$
\begin{aligned}
=(1-\underline{\pi})\left(\sum_{l=\underline{\tau}_{1}}^{\bar{\tau}_{1}-1} \sum_{v=k-l+1}^{k} g^{T}(x(v)) Q_{1} g(x(v))\right. \\
\left.\quad-\sum_{l=\underline{\tau}_{1}}^{\bar{\tau}_{1}-1} \sum_{v=k-l}^{k-1} g^{T}(x(v)) Q_{1} g(x(v))\right)
\end{aligned}
$$$$
=(1-\underline{\pi}) \sum_{l=\underline{\tau}_{1}}^{\bar{\tau}_{1}-1}\left(g^{T}(x(k)) Q_{1} g(x(k))\right.
$$

$$
\left.-g^{T}(x(k-\iota)) Q_{1} g(x(k-\iota))\right)
$$$$
=(1-\underline{\pi})\left(\bar{\tau}_{1}-\underline{\tau}_{1}\right) g^{T}(x(k)) Q_{1} g(x(k))
$$

$$
-(1-\underline{\pi}) \sum_{v=k-\bar{\tau}_{1}+1}^{k-\underline{\tau}_{1}} g^{T}(x(v)) Q_{1} g(x(v))
$$

$\mathbb{E}\left[V_{4}\left(\mathbf{x}_{k+1}, k+1, r(k+1)\right) \mid \mathbf{x}_{k}, r(k)=i\right]-V_{4}\left(\mathbf{x}_{k}, k, i\right)$

$=\sum_{j=1}^{n_{0}} \pi_{i, j} \sum_{l=\tau_{2, j}}^{+\infty} \mu_{\iota} \sum_{v=k-\iota+1}^{k} h^{T}(x(v)) Q_{2} h(x(v))$ 


$$
\begin{aligned}
& -\sum_{\iota=\tau_{2, i}}^{+\infty} \mu_{\iota} \sum_{v=k-\iota}^{k-1} h^{T}(x(v)) Q_{2} h(x(v)) \\
& \leq \sum_{j \neq i} \pi_{i, j}\left(\sum_{\iota=\tau_{2, j}}^{+\infty} \mu_{\iota} \sum_{v=k-\imath+1}^{k-1} h^{T}(x(v)) Q_{2} h(x(v))\right. \\
& \left.-\sum_{\imath=\tau_{2, i}}^{+\infty} \mu_{\iota} \sum_{v=k-\imath+1}^{k-1} h^{T}(x(v)) Q_{2} h(x(v))\right) \\
& +\sigma_{\underline{\tau}_{2}} h^{T}(x(k)) Q_{2} h(x(k)) \\
& -\sum_{\iota=\tau_{2, i}}^{+\infty} \mu_{\iota} h^{T}(x(k-\iota)) Q_{2} h(x(k-\iota)) \\
& \leq \sum_{j \neq i} \pi_{i, j}\left(\sum_{l=\underline{\tau}_{2}}^{+\infty} \mu_{\iota} \sum_{v=k-\imath+1}^{k-1} h^{T}(x(v)) Q_{2} h(x(v))\right. \\
& \left.-\sum_{\imath=\bar{\tau}_{2}}^{+\infty} \mu_{\iota} \sum_{v=k-l+1}^{k-1} h^{T}(x(v)) Q_{2} h(x(v))\right) \\
& +\sigma_{\underline{\tau}_{2}} h^{T}(x(k)) Q_{2} h(x(k)) \\
& -\sum_{\iota=\tau_{2, i}}^{+\infty} \mu_{\iota} h^{T}(x(k-\iota)) Q_{2} h(x(k-\iota)) \\
& \leq(1-\underline{\pi}) \sum_{l=\underline{\tau}_{2}}^{\bar{\tau}_{2}-1} \mu_{\iota} \sum_{v=k-\imath+1}^{k-1} h^{T}(x(v)) Q_{2} h(x(v)) \\
& +\sigma_{\underline{\tau}_{2}} h^{T}(x(k)) Q_{2} h(x(k)) \\
& -\sum_{\iota=\tau_{2, i}}^{+\infty} \mu_{\iota} h^{T}(x(k-\iota)) Q_{2} h(x(k-\iota)) \\
& \leq(1-\underline{\pi}) \widehat{\mu} \sum_{l=\underline{\tau}_{2}}^{\bar{\tau}_{2}-1} \sum_{v=k-l+1}^{k-1} h^{T}(x(v)) Q_{2} h(x(v)) \\
& +\sigma_{\underline{\tau}_{2}} h^{T}(x(k)) Q_{2} h(x(k)) \\
& -\sum_{m=\tau_{2, i}}^{+\infty} \mu_{m} h^{T}(x(k-m)) Q_{2} h(x(k-m)) ; \\
& \mathbb{E}\left[V_{5}\left(\mathbf{x}_{k+1}, k+1, r(k+1)\right) \mid \mathbf{x}_{k}, r(k)=i\right]-V_{5}\left(\mathbf{x}_{k}, k, i\right) \\
& =(1-\underline{\pi}) \widehat{\mu}\left[\sum_{s=\underline{\tau}_{2}}^{\bar{\tau}_{2}-1} \sum_{l=1}^{s-1} \sum_{v=k-l+1}^{k} h^{T}(x(v)) Q_{2} h(x(v))\right. \\
& \left.-\sum_{s=\underline{\tau}_{2}}^{\bar{\tau}_{2}-1} \sum_{\iota=1}^{s-1} \sum_{v=k-\iota}^{k-1} h^{T}(x(v)) Q_{2} h(x(v))\right]
\end{aligned}
$$$$
=(1-\underline{\pi}) \widehat{\mu}\left[\sum _ { s = \underline { \tau } _ { 2 } } ^ { \overline { \tau } _ { 2 } - 1 } \sum _ { l = 1 } ^ { s - 1 } \left(h^{T}(x(k)) Q_{2} h(x(k))\right.\right.
$$

$$
\begin{gathered}
\left.\left.-h^{T}(x(k-\imath)) Q_{2} h(x(k-\iota))\right)\right] \\
=(1-\underline{\pi}) \hat{\mu}\left[\frac{1}{2}\left(\bar{\tau}_{2}-\underline{\tau}_{2}\right)\left(\bar{\tau}_{2}+\underline{\tau}_{2}-3\right) h^{T}(x(k)) Q_{2} h\right. \\
\left.\times(x(k))-\sum_{\imath=\underline{\tau}_{2}}^{\bar{\tau}_{2}-1} \sum_{v=k-l+1}^{k-1} h^{T}(x(v)) Q_{2} h(x(v))\right] .
\end{gathered}
$$

Therefore, we have

$$
\begin{aligned}
& \mathbb{E}\left[V\left(\mathbf{x}_{k+1}, k+1, r(k+1)\right) \mid \mathbf{x}_{k}, r(k)=i\right]-V\left(\mathbf{x}_{k}, k, i\right) \\
& =\sum_{j=1}^{5}\left[\mathbb{E}\left[V_{j}\left(\mathbf{x}_{k+1}, k+1, r(k+1)\right) \mid \mathbf{x}_{k}, r(k)=i\right]\right. \\
& \left.\quad-V_{j}\left(\mathbf{x}_{k}, k, i\right)\right] \\
& \leq \xi^{T}\left(\mathbf{x}_{\mathbf{k}}, i\right) \mathscr{A}_{K}^{T}(i) \bar{P}_{i} \mathscr{A}_{K}(i) \xi\left(\mathbf{x}_{\mathbf{k}}, i\right)-x^{T}(k) P_{i} x(k) \\
& +\alpha_{1} g^{T}(x(k)) Q_{1} g(x(k)) \\
& \quad-g^{T}\left(x\left(k-\tau_{1, i}\right)\right) Q_{1} g\left(x\left(k-\tau_{1, i}\right)\right) \\
& +\alpha_{2} h^{T}(x(k)) Q_{2} h(x(k)) \\
& \quad-\sum_{m=\tau_{2, i}}^{+\infty} \mu_{m} h^{T}(x(k-m)) Q_{2} h(x(k-m)),
\end{aligned}
$$

where $\alpha_{1}=(1-\underline{\pi})\left(\bar{\tau}_{1}-\underline{\tau}_{1}\right)+1, \alpha_{2}=\sigma_{\underline{\tau}_{2}}+(1 / 2) \widehat{\mu}(1-\underline{\pi})\left(\bar{\tau}_{2}-\right.$ $\left.\underline{\tau}_{2}\right)\left(\bar{\tau}_{2}+\underline{\tau}_{2}-3\right)$.

By Lemma 7, it is clear that

$$
\begin{aligned}
& -\sum_{m=\tau_{2, i}}^{+\infty} \mu_{m} h^{T}(x(k-m)) Q_{2} h(x(k-m)) \\
& \quad \leq-\frac{1}{\sigma_{\tau_{2, i}}} \sum_{m=\tau_{2, i}}^{+\infty} \mu_{m} h^{T}(x(k-m)) Q_{2} \sum_{m=\tau_{2, i}}^{+\infty} \mu_{m} h(x(k-m)) .
\end{aligned}
$$

Also, from the conditions (2) and Lemma 8, it follows that

$$
\begin{aligned}
& x^{T}(k) \breve{F}_{1} x(k)-2 x^{T}(k) \breve{F}_{2} f(x(k)) \\
& +f^{T}(x(k)) f(x(k)) \leq 0, \\
& x^{T}(k) \breve{G}_{1} x(k)-2 x^{T}(k) \breve{G}_{2} g(x(k)) \\
& +g^{T}(x(k)) g(x(k)) \leq 0, \\
& x^{T}(k) \breve{H}_{1} x(k)-2 x^{T}(k) \breve{H}_{2} h(x(k)) \\
& +h^{T}(x(k)) h(x(k)) \leq 0 .
\end{aligned}
$$


From (26)-(28), it follows readily that

$$
\begin{aligned}
\mathbb{E} & {\left[V\left(\mathbf{x}_{k+1}, k+1, r(k+1)\right) \mid \mathbf{x}_{k}, r(k)=i\right]-V\left(\mathbf{x}_{k}, k, i\right) } \\
\leq & \xi^{T}\left(\mathbf{x}_{\mathbf{k}}, i\right) \mathscr{A}_{K}^{T}(i) \bar{P}_{i} \mathscr{A}_{K}(i) \xi\left(\mathbf{x}_{\mathbf{k}}, i\right)-x^{T}(k) P_{i} x(k) \\
& +\alpha_{1} g^{T}(x(k)) Q_{1} g(x(k)) \\
& -g^{T}\left(x\left(k-\tau_{1, i}\right)\right) Q_{1} g\left(x\left(k-\tau_{1, i}\right)\right) \\
& +\alpha_{2} h^{T}(x(k)) Q_{2} h(x(k)) \\
& -\frac{1}{\sigma_{\tau_{2, i}}} \sum_{m=\tau_{2, i}}^{+\infty} \mu_{m} h^{T}(x(k-m)) Q_{2} \sum_{m=\tau_{2, i}}^{+\infty} \mu_{m} h^{T}(x(k-m)) \\
& -\left(x^{T}(k) \breve{F}_{1} x(k)-2 x^{T}(k) \breve{F}_{2} f(x(k))\right. \\
& \left.+f^{T}(x(k)) f(x(k))\right) \\
& -\left(x^{T}(k) \breve{G}_{1} x(k)-2 x^{T}(k) \breve{G}_{2} g(x(k))\right. \\
& +x^{T}(k)\left[R_{1}(i)+K^{T}(i)\left[\bar{\Phi}^{T}(k)\right) g(x(k))\right) \\
& \left.+h^{T}(x(k)) h(x(k))\right) \\
& \breve{H}_{1} x(k)-2 x^{T}(k) \breve{H}_{2} h(x(k)) \\
& \\
& \\
& \\
&
\end{aligned}
$$

which, together with (21), implies

$$
\begin{aligned}
& \mathbb{E}\left[V\left(\mathbf{x}_{k+1}, k+1, r(k+1)\right)\right]-\mathbb{E}\left[V\left(\mathbf{x}_{k}, k, r(k)\right)\right] \\
& \leq-\mathbb{E}\left[x^{T}(k)\left(R_{1}(r(k))+K^{T}(r(k)) R_{2} K(r(k))\right) x(k)\right] .
\end{aligned}
$$

Therefore,

$$
\begin{aligned}
& \mathbb{E}\left[V\left(\mathbf{x}_{k+1}, k+1, r(k+1)\right)\right]-\mathbb{E}\left[V\left(\mathbf{x}_{k}, k, r(k)\right)\right] \\
& \quad \leq-\lambda_{0} \mathbb{E}|x(k)|^{2},
\end{aligned}
$$

where $\lambda_{0}=\min _{i \in \mathcal{N}}\left\{\lambda_{\min }\left(R_{1}(i)\right)\right\}$.

Let $s$ be an arbitrary positive integer; then it can be inferred from (31) that

$$
\begin{aligned}
& \mathbb{E}\left[V\left(\mathbf{x}_{s+1}, s+1, r(s+1)\right)\right]-\mathbb{E}\left[V\left(\mathbf{x}_{0}, 0, r(0)\right)\right] \\
& \quad \leq-\lambda_{0} \sum_{k=0}^{s} \mathbb{E}\left[|x(k)|^{2}\right],
\end{aligned}
$$

or

$$
\begin{aligned}
& \mathbb{E}\left[V\left(\mathbf{x}_{s+1}, s+1, r(s+1)\right)\right]-V\left(\mathbf{x}_{0}, 0, r(0)\right) \\
& \leq-\lambda_{0} \sum_{k=0}^{s} \mathbb{E}\left[|x(k)|^{2}\right],
\end{aligned}
$$

which results in

$$
\sum_{k=0}^{s} \mathbb{E}\left[|x(k)|^{2}\right] \leq \frac{1}{\lambda_{0}} V\left(\mathbf{x}_{0}, 0, r(0)\right) .
$$

It can now be concluded that the series $\sum_{k=0}^{+\infty} \mathbb{E}\left[|x(k)|^{2}\right]$ is convergent, and therefore

$$
\lim _{k \rightarrow+\infty} \mathbb{E}\left[|x(k)|^{2}\right]=0
$$

Therefore, the closed-loop system (5a) and (5b) is asymptotically stable in mean square.

On the other hand, for any positive integer $s$, from (30) we have

$$
\begin{aligned}
& \mathbb{E}\left[\sum_{k=0}^{s}\left(x^{T}(k) R_{1}(r(k)) x(k)+u^{T}(k) R_{2}(r(k)) u(k)\right)\right] \\
& =\mathbb{E}\left[\sum_{k=0}^{s} x^{T}(k)\left(R_{1}(r(k))+K^{T}(r(k)) R_{2}(r(k)) K(r(k))\right)\right. \\
& \left.\quad \times x(k)+V\left(\mathbf{x}_{k+1}, k+1, r(k+1)\right)-V\left(\mathbf{x}_{k}, k, r(k)\right)\right] \\
& -\mathbb{E}\left[V\left(\mathbf{x}_{s+1}, s+1, r(s+1)\right)\right]+V\left(\mathbf{x}_{0}, 0, r(0)\right) \\
& \leq V\left(x_{0}, 0, r(0)\right) .
\end{aligned}
$$

Letting $s \rightarrow+\infty$, we have

$$
J \leq V\left(x_{0}, 0, r_{0}\right)
$$

namely, (16) holds. This completes the proof of the theorem.

Theorem 10 provides a sufficient condition to determine if a given controller is a guaranteed cost controller. Next, we turn to the design problem of guaranteed cost controller for the system (2a) and (2b). For this, we have the following results.

Theorem 11. Consider the system (2a) and (2b). If there exist a set of positive definite matrices $X_{i}(i \in \mathcal{N})$, a set of matrices $Y_{i}(i \in \mathcal{N})$, and two positive definite matrices $Q_{1}$ and $Q_{2}$ such that the LMIs (39) hold, then $u(k)=K(r(k)) x(k)$ with $K(i)=Y_{i} X_{i}^{-1}$ is a guaranteed cost controller for the system (2a) and (2b), and the cost function satisfies the following bound: 


$$
\begin{aligned}
& J \leq \gamma=x^{T}(0) X_{r_{0}}^{-1} x(0)+\sum_{v=-\tau_{1, r_{0}}}^{-1} g^{T}(x(v)) Q_{1} g(x(v))+(1-\underline{\pi}) \sum_{l=\underline{\tau}_{1}}^{\bar{\tau}_{1}-1} \sum_{v=-l}^{-1} g^{T}(x(v)) Q_{1} g(x(v)) \\
& +\sum_{\iota=\tau_{2, r_{0}}}^{+\infty} \mu_{\iota} \sum_{v=-\iota}^{-1} h^{T}(x(v)) Q_{2} h(x(v))+(1-\underline{\pi}) \widehat{\mu} \sum_{s=\underline{\tau}_{2}}^{\bar{\tau}_{2}-1} \sum_{l=1}^{s-1} \sum_{v=-\iota}^{-1} h^{T}(x(v)) Q_{2} h(x(v)) ; \\
& \bar{\Phi}(i) \triangleq\left[\begin{array}{cccccc}
-X_{i} & X_{i} \Theta & X_{i} & X_{i} & Y_{i}^{T} & \left(A(i) X_{i}+E(i) Y_{i}\right)^{T} W_{i} \\
\Theta^{T} X_{i} & \Upsilon(i) & 0 & 0 & 0 & \Sigma^{T}(i) W_{i} \\
X_{i} & 0 & \Xi^{-1} & 0 & 0 & 0 \\
X_{i} & 0 & 0 & -R_{1}^{-1}(i) & 0 & 0 \\
Y_{i} & 0 & 0 & 0 & -R_{2}^{-1}(i) & 0 \\
W_{i}^{T}\left(A(i) X_{i}+E(i) Y_{i}\right) & W_{i}^{T} \Sigma(i) & 0 & 0 & 0 & -\mathscr{X}
\end{array}\right]<0
\end{aligned}
$$

where $W_{i}=\left[\sqrt{\pi_{i 1}} I, \sqrt{\pi_{i 2}} I, \ldots, \sqrt{\pi_{i n_{0}}} I\right], \mathscr{X}=$ $\operatorname{diag}\left\{X_{1}, X_{2}, \ldots, X_{n_{0}}\right\}$, and $\Theta, \Upsilon(i), \Xi$ and $\Sigma(i)$ are defined as in Theorem 10.

Proof. Let $P_{i}=X_{i}^{-1}, Y_{i}=K(i) P_{i}^{-1}$, and $\vartheta(i)=\operatorname{diag}\{P_{i}, \overbrace{I, \ldots, I}^{6}\}$. Then, inequality (39) is equivalent to

$$
\vartheta(i) \bar{\Phi}(i) \vartheta(i)<0
$$

namely,

$$
\left[\begin{array}{cccccc}
-P_{i} & \Theta & I & I & K^{T}(i) & A_{K}^{T}(i) W_{i} \\
\Theta^{T} & \Upsilon(i) & 0 & 0 & 0 & \Sigma^{T}(i) W_{i} \\
I & 0 & \Xi^{-1} & 0 & 0 & 0 \\
I & 0 & 0 & -R_{1}^{-1}(i) & 0 & 0 \\
K(i) & 0 & 0 & 0 & -R_{2}^{-1}(i) & 0 \\
W_{i}^{T} A_{K}(i) & W_{i}^{T} \Sigma(i) & 0 & 0 & 0 & -\mathscr{P}^{-1}
\end{array}\right]
$$

$<0$,

where $\mathscr{P}=\operatorname{diag}\left\{P_{1}, P_{2}, \ldots, P_{n_{0}}\right\}$.

From Lemma 9, it follows readily that (41) is equivalent to (21) and is therefore equivalent to (17).

By Theorem 10, $u(k)=K(r(k)) x(k)$ with $K(i)=Y_{i} X_{i}^{-1}$ is a guaranteed cost controller for the system (2a) and (2b), and the cost function satisfies the bound as shown in (38).

Remark 12. In Theorem 11, the bound $\gamma$ of the cost function depends on the parameters $X_{i}, Q_{1}$, and $Q_{2}$ in addition to the initial value of the state and mode of the system. Next, we will design an optimal state-feedback guaranteed cost controller $u(k)=K(r(k)) x(k)$, which minimizes the bound of the guaranteed cost function.

Theorem 13. Consider the system (2a) and (2b) with cost function (4). If the following optimal problem of a linear objective

$$
\min _{\substack{\beta_{0}, X_{i}, Y_{i} \\ Q_{1}, Q_{2}, M_{i}}}\left(\beta_{0}+\operatorname{Tr}\left(M_{1}+M_{2}+M_{3}+M_{4}\right)\right)
$$

subject to LMI constraints

(i) $L M I$

(ii) $\left[\begin{array}{cc}-\beta_{0} & x^{T}(0) \\ x(0) & -X_{r_{0}}\end{array}\right]<0$,

(iii) $\Omega_{k}<0,(k=1,2,3,4)$

has a set of solutions $\beta_{0}, X_{i}(i \in \mathcal{N}), Y_{i}(i \in \mathcal{N}), Q_{1}, Q_{2}$, $M_{k}(k=1,2,3,4)$, then $u(k)=K(r(k)) x(k)$ with $K(i)=$ $Y_{i} X_{i}^{-1}$ is an optimal guaranteed cost controller for the system (2a) and (2b), which minimizes the guaranteed cost (38).

Here,

$$
\begin{array}{ll}
\Omega_{1}=\left[\begin{array}{cc}
-M_{1} & N_{1}^{T} Q_{1} \\
Q_{1} N_{1} & -Q_{1}
\end{array}\right], & \Omega_{2}=\left[\begin{array}{cc}
-M_{2} & N_{2}^{T} Q_{1} \\
Q_{1} N_{2} & -Q_{1}
\end{array}\right], \\
\Omega_{3}=\left[\begin{array}{cc}
-M_{3} & N_{3}^{T} Q_{2} \\
Q_{2} N_{3} & -Q_{2}
\end{array}\right], & \Omega_{4}=\left[\begin{array}{cc}
-M_{4} & N_{4}^{T} Q_{2} \\
Q_{2} N_{4} & -Q_{2}
\end{array}\right],
\end{array}
$$

where $N_{k}(k=1,2,3,4)$ satisfy

$$
\begin{aligned}
& N_{1} N_{1}^{T}=\sum_{v=-\tau_{1, r_{0}}}^{-1} g(x(v)) g^{T}(x(v)), \\
& N_{2} N_{2}^{T}=(1-\underline{\pi}) \sum_{\imath=\underline{\tau}_{1}}^{\bar{\tau}_{1}-1} \sum_{v=-\iota}^{-1} g(x(v)) g^{T}(x(v)), \\
& N_{3} N_{3}^{T}=\sum_{\iota=\tau_{2, r_{0}}} \mu_{\iota} \sum_{v=-\iota}^{-1} h(x(v)) h^{T}(x(v)), \\
& N_{4} N_{4}^{T}=(1-\underline{\pi}) \widehat{\mu} \sum_{s=\underline{\tau}_{2}} \sum_{l=1}^{s} \sum_{v=-\iota}^{-1} h(x(v)) h^{T}(x(v)) .
\end{aligned}
$$

Proof. According to Theorem 11, $u(k)=K(r(k)) x(k)$ with $K(i)=Y_{i} X_{i}^{-1}$ is a guaranteed cost controller for the system (2a) and (2b) if LMI (39) has a set of solutions $X_{i}, Y_{i}, Q_{1}$, and $Q_{2}$. 


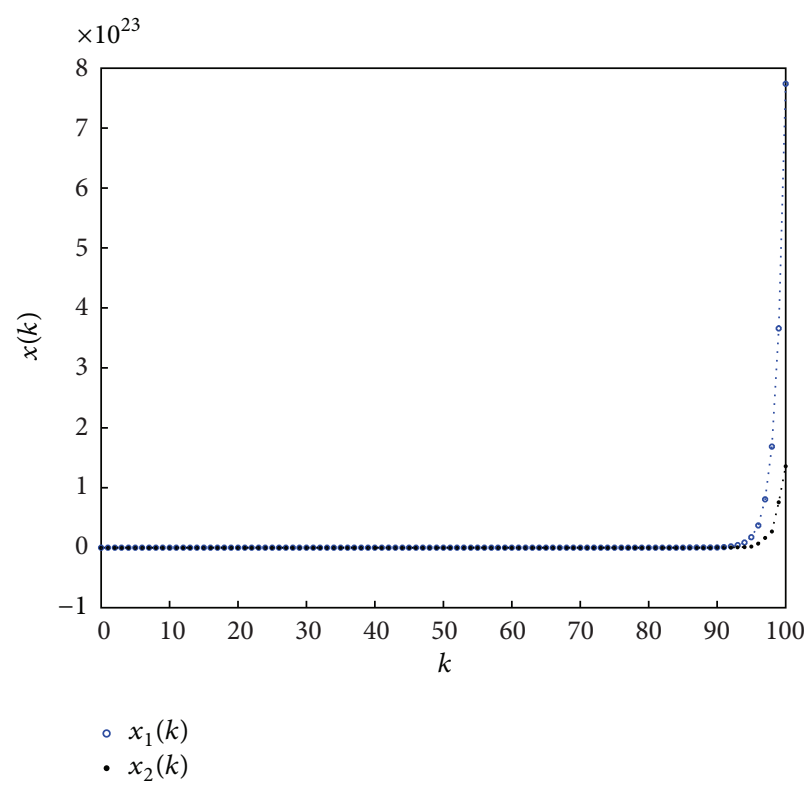

(a) The state evolution of the unforced system

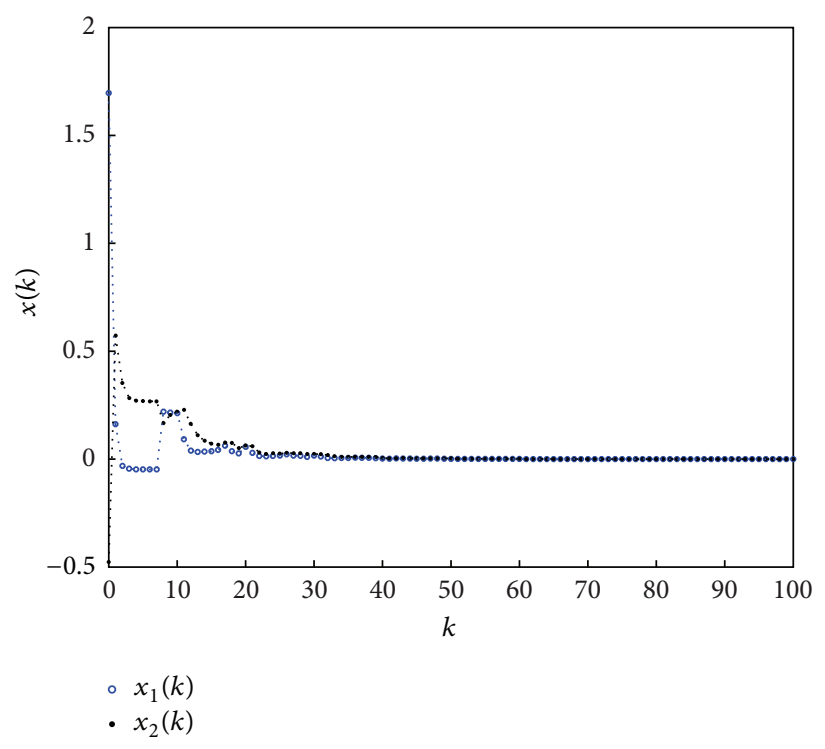

(b) The state evolution of the closed-loop system

FIGURE 1: The comparison of state trajectories of the unforced and the controlled systems.

On the other hand, by Lemma 9 , the inequality in (ii) is equivalent to $x^{T}(0) X_{r_{0}}^{-1} x(0)<\beta_{0}$. Notice that $\Omega_{1}<0$ is equivalent to

$$
\begin{aligned}
& N_{1}^{T} Q_{1} N_{1}<M_{1}, \\
& \sum_{v=-\tau_{1, r_{0}}}^{-1} g^{T}(x(v)) Q_{1} g(x(v)) \\
& =\sum_{v=-\tau_{1, r_{0}}}^{-1} \operatorname{Tr}\left(g^{T}(x(v)) Q_{1} g(x(v))\right) \\
& =\sum_{v=-\tau_{1, r_{0}}}^{-1} \operatorname{Tr}\left(g(x(v)) g^{T}(x(v)) Q_{1}\right) \\
& =\operatorname{Tr}\left(\sum_{v=-\tau_{1, r_{0}}}^{-1} g(x(v)) g^{T}(x(v)) Q_{1}\right) \\
& =\operatorname{Tr}\left(N_{1} N_{1}^{T} Q_{1}\right) \\
& =\operatorname{Tr}\left(N_{1}^{T} Q_{1} N_{1}\right) \quad \text { (Thanks to (46)) } \\
& <\operatorname{Tr}\left(M_{1}\right) .
\end{aligned}
$$

Similarly, from $\Omega_{k}<0 \quad(k=2,3,4)$ it follows that

$$
\begin{gathered}
(1-\underline{\pi}) \sum_{l=\underline{\tau}_{1}}^{\bar{\tau}_{1}-1} \sum_{v=-\iota}^{-1} g(x(v)) g^{T}(x(v))<\operatorname{Tr}\left(M_{2}\right), \\
\sum_{l=\tau_{2, r_{0}}}^{+\infty} \mu_{\iota} \sum_{v=-l}^{-1} h(x(v)) h^{T}(x(v)) \leq \operatorname{Tr}\left(M_{3}\right),
\end{gathered}
$$

$$
(1-\underline{\pi}) \widehat{\mu} \sum_{s=\underline{\tau}_{2}}^{\bar{\tau}_{2}-1} \sum_{l=1}^{s-1} \sum_{v=-l}^{-1} h(x(v)) h^{T}(x(v)) \leq \operatorname{Tr}\left(M_{4}\right)
$$

Accordingly, it follows that $\gamma<\lambda_{0}+\operatorname{Tr}\left(M_{1}+M_{2}+M_{3}+M_{4}\right)$, where $\gamma$ is defined in (38). Since the optimal problem (42) has a set of solutions, the minimization of the guaranteed cost for the system (2a) and (2b) follows from the minimization of $\lambda_{0}+\operatorname{Tr}\left(M_{1}+M_{2}+M_{3}+M_{4}\right)$. The proof of this theorem is completed.

\section{A Numerical Example}

In this section, an example is presented to demonstrate the effectiveness of our main results.

Example 1. For simplicity, consider a two-dimensional system $(2 \mathrm{a})$ and $(2 \mathrm{~b})$ with probability transition matrix $\Pi=$ $\left[\begin{array}{llll}0.6 & 0.3 & 0.3 \\ 0.1 & 0.6 & 0.3 \\ 0.1 & 0.1 & 0.8\end{array}\right]$, and the following matrix parameters:

$$
\begin{array}{ll}
A(1)=\left[\begin{array}{ll}
1.2 & 0.2 \\
0.2 & 0.2
\end{array}\right], & B(1)=\left[\begin{array}{cc}
0.3 & 0.3 \\
0.2 & -0.2
\end{array}\right], \\
C(1)=\left[\begin{array}{cc}
-0.2 & 0.3 \\
0.2 & 0.3
\end{array}\right], & D(1)=\left[\begin{array}{cc}
0.2 & -0.2 \\
0.2 & 0.2
\end{array}\right], \\
E(1)=\left[\begin{array}{l}
1 \\
0
\end{array}\right], & \\
A(2)=\left[\begin{array}{ll}
1.5 & 0.3 \\
0.1 & 0.2
\end{array}\right], & B(2)=\left[\begin{array}{ll}
0.3 & 0.2 \\
0.3 & 0.2
\end{array}\right], \\
C(2)=\left[\begin{array}{ll}
0.4 & 0.3 \\
0.2 & 0.2
\end{array}\right], & D(2)=\left[\begin{array}{ll}
0.3 & 0.2 \\
0.2 & 0.3
\end{array}\right],
\end{array}
$$




$$
\begin{array}{ll}
E(2)=\left[\begin{array}{c}
1.5 \\
0
\end{array}\right], & \\
A(3)=\left[\begin{array}{cc}
2 & 0.1 \\
0.2 & 0.3
\end{array}\right], & B(3)=\left[\begin{array}{ll}
0.2 & 0.2 \\
0.3 & 0.2
\end{array}\right], \\
C(3)=\left[\begin{array}{cc}
0.2 & 0.3 \\
0 & 0.2
\end{array}\right], & D(3)=\left[\begin{array}{cc}
0.2 & 0 \\
0.2 & 0.3
\end{array}\right], \\
E(3)=\left[\begin{array}{c}
1.2 \\
0
\end{array}\right] .
\end{array}
$$

In addition, the parameters for time delays are listed as $\tau_{1,1}=$ $6, \tau_{1,2}=8, \tau_{1,3}=9, \tau_{2,1}=9, \tau_{2,2}=8, \tau_{2,3}=10$, and the initial mode of Markov chain is $r_{0}=1$, and the initial value of the system is $x(m)=(1.6969,-0.4770)^{T}$ for $m \in(-\infty, 0]$, which is stochastically produced by Matlab.

Also, the nonlinear functions are taken as

$$
\begin{aligned}
f(x)= & g(x)=h(x) \\
= & \left(0.1 x_{1}+0.2 x_{2}+0.4 x_{1} \sin x_{2}, 0.3 x_{1}\right. \\
& \left.\quad+0.1 x_{2}+0.4 x_{2} \cos x_{1}\right)^{T} .
\end{aligned}
$$

It can also be seen from (50) that

$$
\breve{F}_{1}=\left[\begin{array}{cc}
-0.0600 & 0.0500 \\
0.0500 & -0.1100
\end{array}\right], \quad \breve{F}_{2}=\left[\begin{array}{ll}
0.1000 & 0.3000 \\
0.2000 & 0.1000
\end{array}\right] .
$$

With the previous parameters, based on Theorem 13 and by using Matlab LMI Toolbox, we solve the linear objective minimization problem (42) and obtain the feasible solutions for $X_{i}, Y_{1}, Q_{1}, Q_{2}, \beta_{0}$, and $M_{i}$ (the values are omitted for space saving). Here, we just give the corresponding optimal control gain matrices $K(1)=-[1.2941,0.3499]$, $K(2)=-[1.1552,0.3687], K(3)=-[1.7817,0.2616]$, and the minimal upper bound $\gamma=5.3853$ of the guaranteed cost. Moreover, the dynamical comparison between the unforced system and closed-loop system is shown in Figure 1.

\section{Conclusions}

In this paper, we have dealt with the guaranteed cost control problem for a class of nonlinear discrete-time systems with Markovian jumping parameters and mode-dependent mixed time delays. The sufficient conditions for the existence of guaranteed cost controllers are established for the system under consideration and related cost function. Furthermore, an LMI-based approach to design the optimal guaranteed cost controller has been formulated to minimize the guaranteed cost of the closed-loop system. A numerical example is also given to illustrate the effectiveness of the proposed methods. It will be interesting to extend the present results to more general cases, for example, the case where the quantized state-feedback is used for stabilization of the system concerned, and the case when the nonlinear stochastic systems are considered with missing measurements. And it would be one of the future research topics.

\section{Acknowledgments}

This work was supported in part by the International Travel Grant 2011/R3 sponsored by the Royal Society of the U.K., the National Natural Science Foundation of China under Grant 61074129, and the Natural Science Foundation of Jiangsu Province of China under Grant BK2012682.

\section{References}

[1] Y.-Y. Cao, J. Lam, and L. Hu, "Delay-dependent stochastic stability and $H_{\infty}$ analysis for time-delay systems with Markovian jumping parameters," Journal of the Franklin Institute. Engineering and Applied Mathematics, vol. 340, no. 6-7, pp. 423434, 2003.

[2] S. S. L. Chang and T. K. C. Peng, "Adaptive guaranteed cost control of systems with uncertain parameters," IEEE Transactions on Automatic Control, vol. 17, no. 4, pp. 474-483, 1972.

[3] D. Ding, Z. Wang, B. Shen, and H. Shu, " $H_{\infty}$ state estimation for discrete-time complex networks with randomly occurring sensor saturations and randomly varying sensor delays," IEEE Transactions on Neural Networks and Learning Systems, vol. 23, no. 5, pp. 725-736, 2012.

[4] D. Ding, Z. Wang, H. Dong, and H. Shu, "Distributed $\mathscr{H}_{\infty}$ state estimation with stochastic parameters and nonlinearities through sensor networks: the finite-horizon case," Automatica, vol. 48, no. 8, pp. 1575-1585, 2012.

[5] H. Dong, Z. Wang, and H. Gao, "Distributed filtering for a class of time-varying systems over sensor networks with quantization errors and successive packet dropouts," IEEE Transactions on Signal Processing, vol. 60, no. 6, pp. 3164-3173, 2012.

[6] H. Dong, Z. Wang, and H. Gao, "Fault detection for Markovian jump systems with sensor saturations and randomly varying nonlinearities," IEEE Transactions on Circuits and Systems. I. Regular Papers, vol. 59, no. 10, pp. 2354-2362, 2012.

[7] J. Hu, Z. Wang, B. Shen, and H. Gao, "Gain-constrained recursive filtering with stochastic nonlinearities and probabilistic sensor delays," IEEE Transactions on Signal Processing, vol. 61, no. 5, pp. 1230-1238, 2013.

[8] J. Hu, Z. Wang, H. Gao, and L. K. Stergioulas, "Robust sliding mode control for discrete stochastic systems with mixed time delays, randomly occurring uncertainties, and randomly occurring nonlinearities," IEEE Transactions on Industrial Electronics, vol. 59, no. 7, pp. 3008-3015, 2012.

[9] Z. Lin and G. Duan, "Robust guaranteed cost control for discrete-time uncertain systems with delay," IEE ProceedingsControl Theory and Application, vol. 146, no. 6, pp. 598-602, 1999.

[10] Y. Liu, Z. Wang, and X. Liu, "Robust $H_{\infty}$ control for a class of nonlinear stochastic systems with mixed time delay," International Journal of Robust and Nonlinear Control, vol. 17, no. 16, pp. 1525-1551, 2007.

[11] B. Shen, Z. Wang, and X. Liu, "Sampled-data synchronization control of dynamical networks with stochastic sampling," IEEE Transactions on Automatic Control, vol. 57, no. 10, pp. 26442650, 2012.

[12] Z. Wang, D. W. C. Ho, H. Dong, and H. Gao, "Robust $\mathscr{H}_{\infty}$ finite-horizon control for a class of stochastic nonlinear timevarying systems subject to sensor and actuator saturations," IEEE Transactions on Automatic Control, vol. 55, no. 7, pp. 1716$1722,2010$. 
[13] Z. Wang, F. Yang, D. W. C. Ho, and X. Liu, "Robust $H_{\infty}$ control for networked systems with random packet losses," IEEE Transactions on Systems, Man, and Cybernetics, Part B, vol. 37, no. 4, pp. 916-924, 2007.

[14] Z. Wang, B. Shen, H. Shu, and G. Wei, "Quantized $H_{\infty}$ control for nonlinear stochastic time-delay systems with missing measurements," IEEE Transactions on Automatic Control, vol. 57, no. 6, pp. 1431-1444, 2012.

[15] L. Yu and J. Chu, "An LMI approach to guaranteed cost control of linear uncertain time-delay systems," Automatica, vol. 35, no. 6, pp. 1155-1159, 1999.

[16] E. K. Boukas and Z. K. Liu, "Robust stability and stabilizability of Markov jump linear uncertain systems with mode-dependent time delays," Journal of Optimization Theory and Applications, vol. 109, no. 3, pp. 587-600, 2001.

[17] E.-K. Boukas and Z. Liu, Deterministic and Stochastic TimeDelay Systems, Springer, New York, NY, USA, 2002.

[18] I. R. Petersen and D. C. McFarlane, "Optimal guaranteed cost control and filtering for uncertain linear systems," IEEE Transactions on Automatic Control, vol. 39, no. 9, pp. 1971-1977, 1994.

[19] S. O. R. Moheimani and I. R. Petersen, "Optimal quadratic guaranteed cost control of a class of uncertain time-delay systems," IEE Proceedings-Control Theory and Applications, vol. 144, no. 2, pp. 183-188, 1997.

[20] S. Boyd, L. El Ghaoui, E. Feron, and V. Balakrishnan, Linear Matrix Inequalities in System and Control Theory, vol. 15 of SIAM Studies in Applied Mathematics, SIAM, Philadelphia, Pa, USA, 1994.

[21] W.-H. Chen, J.-X. Xu, and Z.-H. Guan, "Guaranteed cost control for uncertain Markovian jump systems with mode-dependent time-delays," IEEE Transactions on Automatic Control, vol. 48, no. 12, pp. 2270-2277, 2003.

[22] Z. Zuo, Z. Jia, Y. Wang, H. Zhao, and G. Zhang, "Guaranteed cost control for discrete-time uncertain systems with saturating actuators," in American Control Conference Westin Seattle Hotel, Seattle, Wash, USA, June 2008.

[23] J. Hu, Z. Wang, H. Gao, and L. K. Stergioulas, "Probabilityguaranteed $H_{\infty}$ finite-horizon filtering for a class of nonlinear time-varying systems with sensor saturations," Systems \& Control Letters, vol. 61, no. 4, pp. 477-484, 2012.

[24] J. Hu, Z. Wang, B. Shen, and H. Gao, "Quantized recursive filtering for a class of nonlinear systems with multiplicative noises and missing measurements," International Journal of Control, vol. 86, no. 4, pp. 650-663, 2013.

[25] Y. Liu, Z. Wang, J. Liang, and X. Liu, "Synchronization and state estimation for discrete-time complex networks with distributed delays," IEEE Transactions on Systems, Man, and Cybernetics, Part B, vol. 38, no. 5, pp. 1314-1325, 2008. 


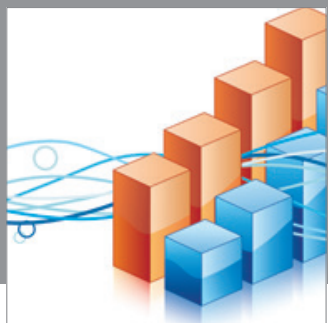

Advances in

Operations Research

mansans

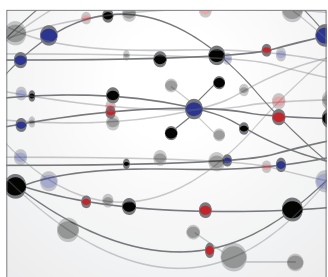

The Scientific World Journal
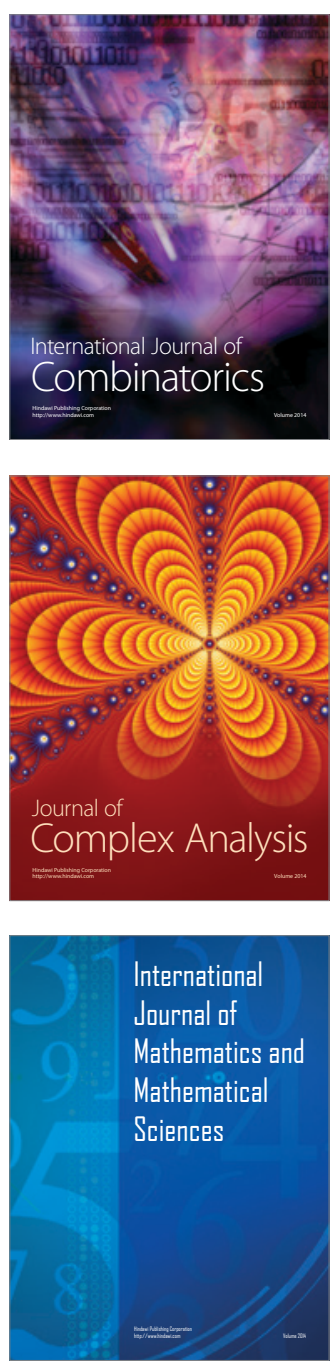
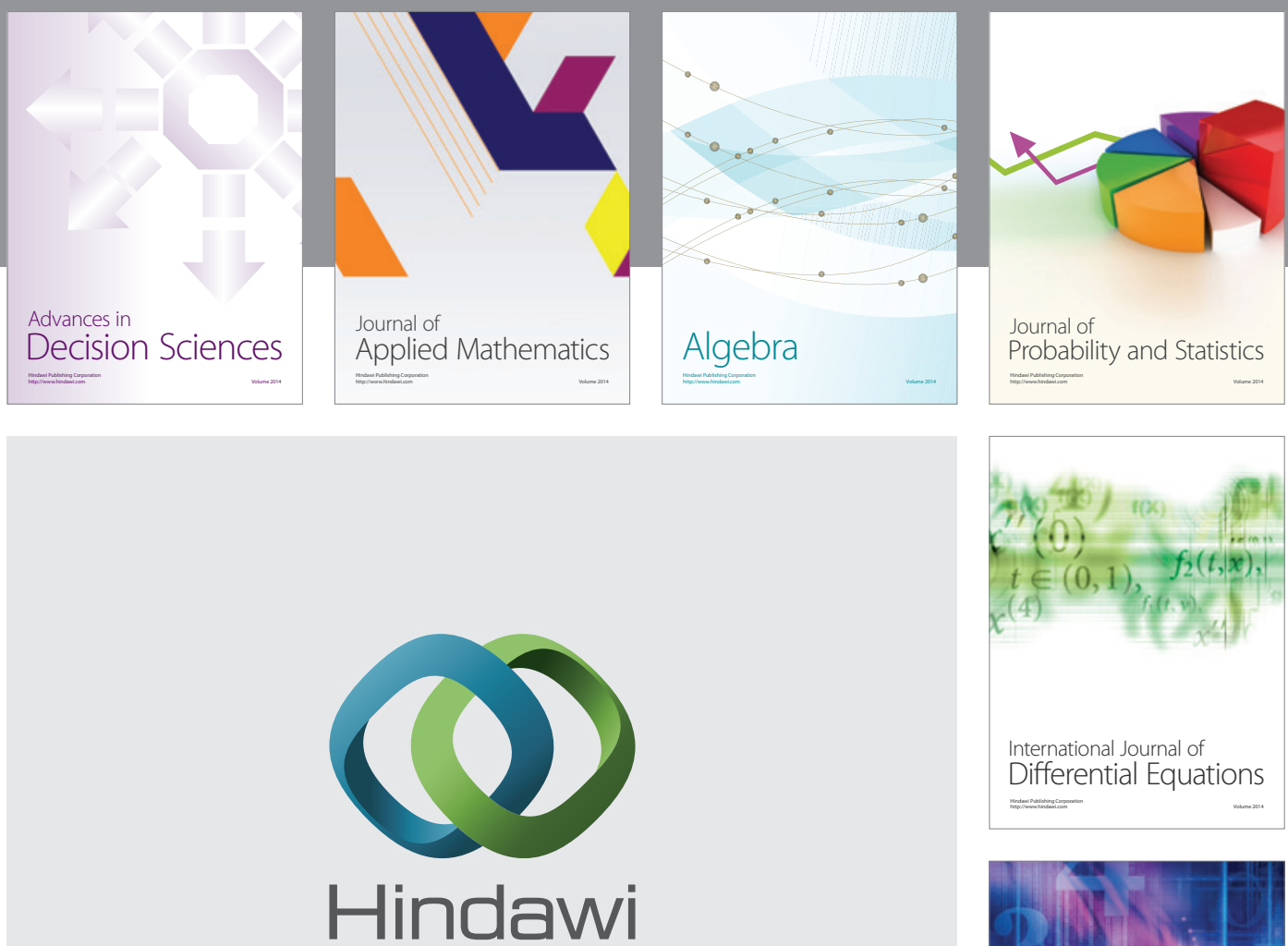

Submit your manuscripts at http://www.hindawi.com
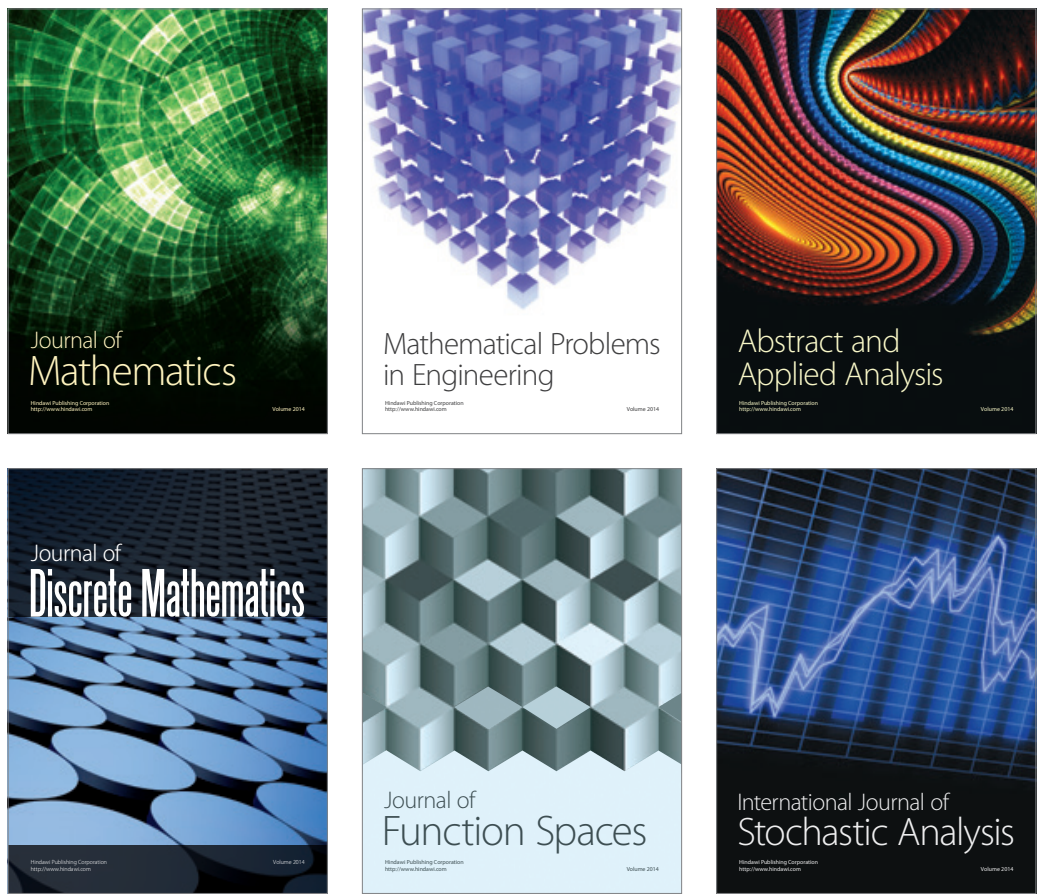

Journal of

Function Spaces

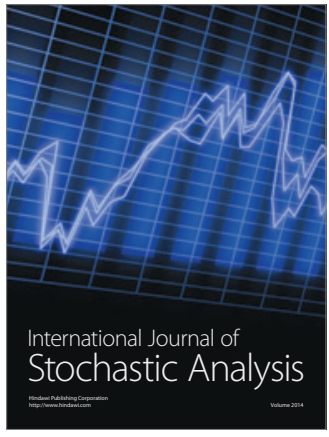

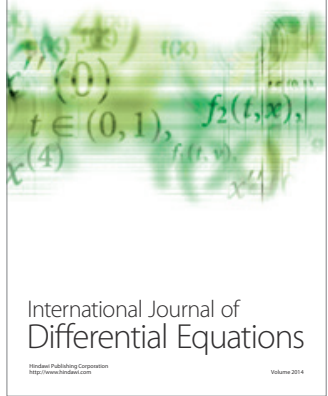
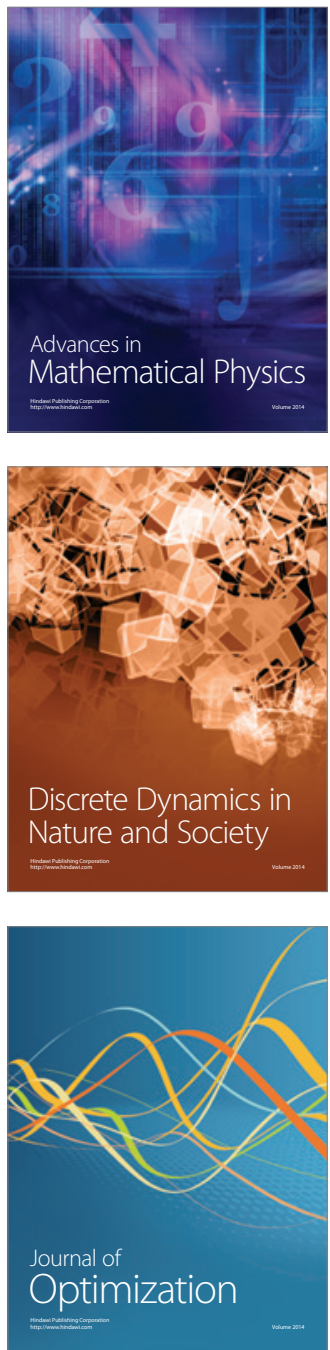